\title{
Wwp2 mediates Oct4 ubiquitination and its own auto-ubiquitination in a dosage-dependent manner
}

Bing Liao ${ }^{1,2,3}$, Ying Jin ${ }^{1,2,4}$

${ }^{1}$ Key Laboratory of Stem Cell Biology, Institute of Health Sciences, Shanghai Institutes for Biological Sciences, Chinese Academy of Sciences/Shanghai Jiao Tong University School of Medicine; 225 South Chongqing Road, Shanghai 200025, China; ${ }^{2}$ Shanghai Stem Cell Institute, Shanghai Jiao Tong University School of Medicine, Shanghai 200025, China; ${ }^{3}$ Graduate School of Chinese Academy of Sciences, Beijing 100000, China; ${ }^{4}$ Key Laboratory of Cell Differentiation and Apoptosis of Chinese Ministry of Education, Shanghai Jiao Tong University School of Medicine, Shanghai 200025, China

Transcription factor Oct4 plays critical roles in maintaining pluripotency and controlling lineage commitment of embryonic stem cells (ESCs). Our previous study indicates that Wwp2, a mouse HECT-type E3 ubiquitin ligase, ubiquitinates Oct4 and promotes its degradation in a heterologous system. However, roles of Wwp2 in regulating endogenous Oct4 protein levels as well as molecular characteristics of the function of Wwp2 have not been determined. Here, we report that Wwp2 plays an important role in Oct4 ubiquitination and degradation during differentiation of embryonal carcinoma cells (ECCs), although it does not appear to affect Oct4 protein levels in the undifferentiated ECCs and ESCs. Importantly, inhibition of Wwp2 expression by specific RNA interference elevates the Oct4 protein level, leading to attenuation in retinoid acid-induced activation of differentiation-related marker genes. Mechanistically, Wwp2 catalyzes Oct4 poly-ubiquitination via the lysine 63 linkage in a dosage-dependent manner. Interestingly, Wwp2 also regulates its own ligase activity in a similar manner. Moreover, auto-ubiquitination of Wwp2 occurs through an intra-molecular mechanism. Taken together, these results demonstrate a crucial role of Wwp2 in controlling endogenous Oct4 protein levels during differentiation processes of ECCs and suggest an interesting dosagedependent mechanism for regulating the catalytic activity of the E3 ubiquitin ligase, Wwp2.

Keywords: degradation, embryonal carcinoma cells, Oct4, auto-ubiquitination, ubiquitin chain linkage, Wwp2

Cell Research (2010) 20:332-344. doi: 10.1038/cr.2009.136; published online 8 December 2009

\section{Introduction}

The POU transcription factor Oct4, an essential regulator in mammalian embryonic development, is highly expressed in cultured embryonic cell lines, including embryonic stem cells (ESCs), embryonal carcinoma cells (ECCs) and embryonic germ cells (EGCs), and is absent from all of the differentiated somatic cell types [1-3]. Uniquely, the precise level of Oct4 determines different fates of ESCs: a more than two-fold elevation induces differentiation toward mesodermal and primitive endodermal lineages, while repression of Oct4 expression

\footnotetext{
Correspondence: Ying Jin

Tel/Fax: +86-21-63852591

E-mail: yjin@sibs.ac.cn

Received 8 May 2009; revised 4 September 2009; accepted 21 September 2009; published online 8 December 2009
}

causes differentiation into trophectodermal lineages [4] Thus, an accurately controlled Oct4 level is important not only for self-renewal of undifferentiated ESCs but also for their differentiation processes. Recently, Yamanaka and colleagues showed that ectopic expression of four factors (Oct4, Sox2, Klf4 and c-Myc) can reprogram differentiated somatic cells into pluripotent stem cells (induced pluripotent stem cells) [5]. More recently, it was reported that Oct4 alone is sufficient to induce pluripotency in adult neural stem cells, further highlighting the central position of Oct4 in stem cell biology [6]. One critical unsolved issue is how the expression level of Oct4 is controlled in undifferentiated ESCs as well as during their differentiation. Although regulation of Oct4 expression at the transcriptional level has been extensively investigated, roles of post-transcriptional modifications in Oct4-mediated functions are poorly explored.

Post-translational modification of cellular proteins 
by ubiquitin $(\mathrm{Ub})$ regulates diverse biological processes ranging from protein degradation, endocytosis of membrane proteins, protein-protein interactions in signal transduction, to cell-cycle progression, apoptosis, gene transcription and immune responses [7-10]. Ubiquitination, covalent conjugation of $\mathrm{Ub}$ to client proteins, requires a sequential action of three enzymes: Ubactivating enzyme (E1), which activates Ub in an ATPdependent manner; the activated $\mathrm{Ub}$ is then transferred to the active cysteine residue of a Ub-conjugating enzyme (E2), and a Ub-protein ligase (E3), which specifically recognizes substrates and binds to an E2, facilitating the transfer of $\mathrm{Ub}$ from E2 to a lysine (Lys) residue of the target protein to form an isopeptide bond. There are mainly two distinct E3 families: one is the RING finger E3 family, and the other is the HECT (homologous to E6-AP carboxyl-terminus)-type E3 family. One subfamily of the HECT-type E3 ligases is characterized by containing three modular domains: an N-terminal C2 domain involved in membrane interaction and subcellular localization [11], a C-terminal HECT domain recruiting the E2 and possessing the enzymatic E3 activity [12] and 2-4 tryptophan-based WW domains in the middle mediating the specific interaction with substrates generally containing proline-rich peptide motifs [13-16].

Conjugation of $\mathrm{Ub}$ to substrates can be of poly-ubiquitination with a polymeric Ub chain or of monoubiquitination with a Ub monomer at one or multiple sites. Moreover, there are seven Lys residues (Lys 6, Lys 11, Lys 27, Lys 29, Lys 33, Lys 48 and Lys 63) within Ub, so seven putative types of the isopeptide linkage could exist. In addition, poly-Ub chains could be homogenous (containing a single kind of linkage) or heterogeneous (containing more than one kind of linkage) [17-21]. It is generally accepted that different Ub linkages could lead to various conformations of Ub chains and generate diverse molecular signals in cells. To date, the most well-studied Ub linkages are Lys 48- and Lys 63-linked chains: Lys 48-linked Ub chains usually serve as a signal for proteasomal destruction [22], whereas Lys 63-linked $\mathrm{Ub}$ chains may play a non-proteolytic role in the regulation of transcriptional activities, receptor trafficking, DNA repair and NF-kappaB activation [23-25]. However, emerging evidence suggests that the Lys 63-linked chain might also be implicated in proteasomal degradation of some proteins [26, 27].

In addition to catalyzing ubiquitination of specific substrates, some HECT-type E3 ligases promote their own ubiquitination, which occurs through an inter-molecular or intra-molecular reaction [28], and is governed by post-translational modifications or by interaction with adaptor proteins [10]. Although auto-ubiquitination is generally thought to modulate the abundance of E3 ligases by degradation $[29,30]$, non-proteolytic functions of E3 ligase auto-ubiquitination have also been reported. For example, auto-ubiquitination of Nedd4/Rsp5p-family members was shown to be a critical determinant for their ability to modify $\mathrm{Ub}$ receptor proteins $[31,32]$.

Our previous study has demonstrated that Wwp2 is a HECT-type E3 ligase, highly expressed in undifferentiated ESCs and ECCs. Its expression decreases along with the differentiation process in these cells. Furthermore, Wwp2 promotes ubiquitination of Oct 4 and suppresses the transactivation activity of Oct4 in HEK 293 cells [33]. However, a number of important questions remain unsolved, such as how Wwp2 ubiquitinates Oct4 at the molecular level. Is endogenously expressed Oct4 ubiquitinated by Wwp2? Does Wwp2-mediated Oct4 ubiquitination play any role in controlling the Oct4 protein level in ESCs? In the present study, we report that inhibition of $W w p 2$ expression by specific RNA interference (RNAi) elevates the Oct4 protein level and impairs the differentiation process. Interestingly, Wwp2 regulates its own ligase activity and Oct4 ubiquitination in a dosage-dependent manner during differentiation of ECCs. Moreover, auto-ubiquitination of Wwp2 occurs through an intramolecular mechanism, and is closely associated with its E3 ligase activity. Our results show that Wwp2-mediated Lys 63-linked poly-Ub chains on Oct4 could promote its degradation by the $26 \mathrm{~S}$ proteasome and provide important insights into molecular mechanisms of regulation of Wwp2 ligase activities and Oct4 protein levels during differentiation of ECCs.

\section{Results}

Wwp2-catalyzed Oct4 poly-ubiquitination is linked by Lys 63 and mediates Oct4 degradation through the $26 \mathrm{~S}$ proteasome

To characterize the Wwp2-catalyzed Ub modification on Oct4, we began with identifying the type of Ub chain linkages, as the distinct linkage of poly-Ub chains has been implicated in determining the mode of protein regulation $[34,35]$. An in vitro ubiquitination assay was conducted utilizing serial purified Ub mutants, in which one of the seven Lys $(\mathrm{K})$ was replaced by arginine $(\mathrm{R})$ : K6R-Ub, K11R-Ub, K27R-Ub, K29R-Ub, K33R-Ub, K48R-Ub and K63R-Ub. As shown in Figure 1A, polyubiquitination of Oct4 was eliminated when K63R-Ub was used in the reaction mixture (lane 7), whereas other mutants did not affect the modification of Oct 4 by polyUb. The finding suggests that the Wwp2-catalyzed poly$\mathrm{Ub}$ modification of Oct4 is dependent on the presence of the Lys 63-linkage. 

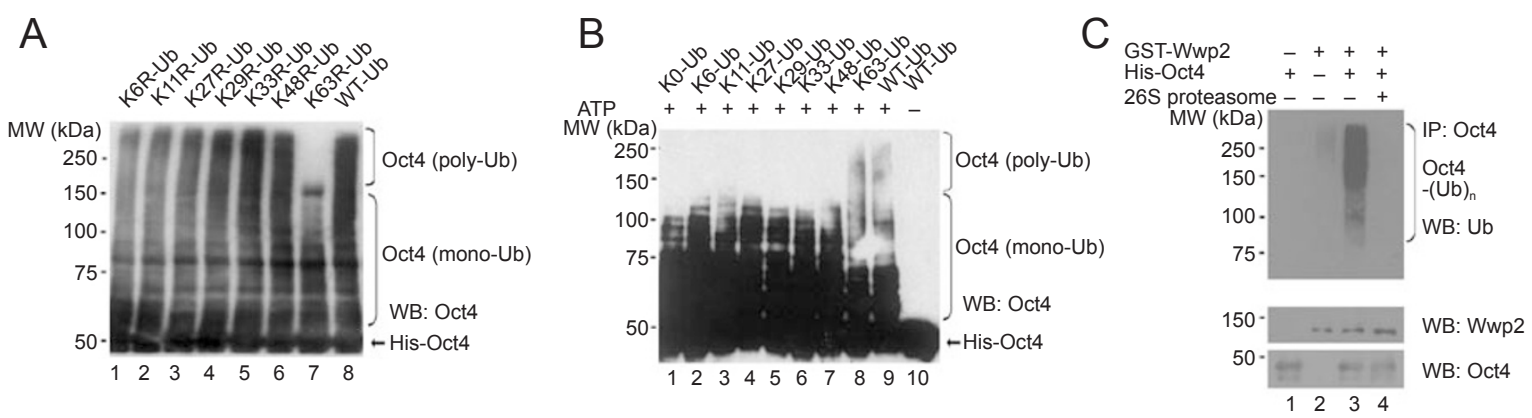

Figure 1 Wwp2-mediated Oct4 poly-ubiquitination occurs through the Lys 63 linkage in vitro. (A) In vitro ubiquitination assays were carried out using each of the seven Lys mutants or wild-type Ub. Western blot (WB) analysis was done with Oct4 antibody (C70). (B) The same reactions were performed except that only one lysine (Lys) residue was present in Ub mutants. K0-Ub: no Lys residue in Ub. (C) Ubiquitinated Oct4 is disassembled by $26 \mathrm{~S}$ proteasomes in vitro. After immunoprecipitation with Oct4 antibody (N124), western blot analysis of in vitro ubiquitination reaction products with a Ub antibody was conducted (P4D1) (top panel). Protein levels of GST-Wwp2 and His-Oct4 were determined in the reaction product by western blotting using Wwp2 antibody and Oct4 antibody (N124), respectively.

To determine whether the Lys 63 residue is sufficient to support a full extent of ubiquitination of Oct4, we performed an in vitro ubiquitination assay with a panel of $\mathrm{Ub}$ mutants, in which only one of the seven Lys residues remained: K6-Ub, K11-Ub, K27-Ub, K29-Ub, K33-Ub, $\mathrm{K} 48-\mathrm{Ub}$ and K63-Ub. As shown in Figure 1B, Wwp2catalyzed ubiquitinated Oct4 at higher molecular weights was only observed in the presence of K63-Ub (lane 8) at a level comparable to that in the presence of wild-type (WT) Ub (lane 9), indicating that the Lys 63 residue is sufficient for Wwp2-mediated poly-Ub chain formation on Oct4. Our results argue that Wwp2-mediated Oct4 poly-Ub chains are homogenously linked through the Lys 63 residue of the $\mathrm{Ub}$ molecule.

Our previous study showed that Wwp2-mediated ubiquitination might target Oct4 degradation through the 26S proteasome in HEK 293 cells [33]. However, the poly-Ub chain linked by Lys 63 is generally not considered as a degradation signal [34]. To validate that Wwp2catalyzed ubiquitinated Oct 4 can be degraded through the $\mathrm{Ub}-26 \mathrm{~S}$ proteasome pathway, we conducted a $26 \mathrm{~S}$ proteasome degradation assay using purified His-Oct4. As illustrated in Figure 1C, His-Oct4 fusion proteins were modified by Ub (top panel, lane 3). Strikingly, the ubiquitinated His-Oct4 was abrogated when $26 \mathrm{~S}$ proteasomes were added to the reaction mixture (top panel, lane 4), providing biochemical evidence that Wwp2-mediated ubiquitination of Oct4 can be disassembled by the $26 \mathrm{~S}$ proteasome. Furthermore, the total protein level of HisOct4 decreased after $26 \mathrm{~S}$ proteasome addition (bottom panel, compare lane 4 to lane 3). Thus, our data support the notion that Wwp2-catalyzed Oct4 poly-ubiquitination is linked by the Lys 63 residue and the modified Oct4 is subject to degradation in the $26 \mathrm{~S}$ proteasome.

\section{Wwp2 regulates Oct4 protein levels during differentia- tion of ECCS}

It is necessary now to examine whether endogenous Oct4 is ubiquitinated and degraded. Western blot analysis detected protein bands at higher molecular weights indicative of attachment of poly-Ub to endogenous Oct4 in CGR8 mouse ESCs (Figure 2A, lane 2). Similar result was obtained when the western blot was analyzed by a Ub antibody, verifying that those bands at the higher molecular weights were indeed Ub-modified Oct4 (lane 4). Of note, these Ub-modified Oct4 signals were not detected if the cells were not pretreated with the proteasome inhibitor MG132 (data not shown), suggesting that ubiquitinated Oct4 in ESCs could be degraded through the $26 \mathrm{~S}$ proteasome.

The next question is whether Wwp2 plays any role in Oct4 ubiquitination and degradation in ESCs. To search for the answer, we knocked down $W w p 2$ expression by an RNAi approach in CGR8 cells. Disappointedly, we did not find significant differences in the steady-state level of Oct4 proteins between control cells and $W w p 2$ RNAi-treated cells (data not shown). It appears that Wwp2 may not be the major Ub E3 ligase responsible for Oct4 degradation in undifferentiated ESCs. On the other hand, it has been well established that Oct4 expression is rapidly down-regulated during ESC differentiation. A previous study reported that the protein level of Oct4 is significantly down-regulated, while the Oct4 transcript is not changed during the process of primitive endoderm differentiation [36]. Therefore, we generated a stable F9 mouse ECC line expressing His-tagged Ub for 
A
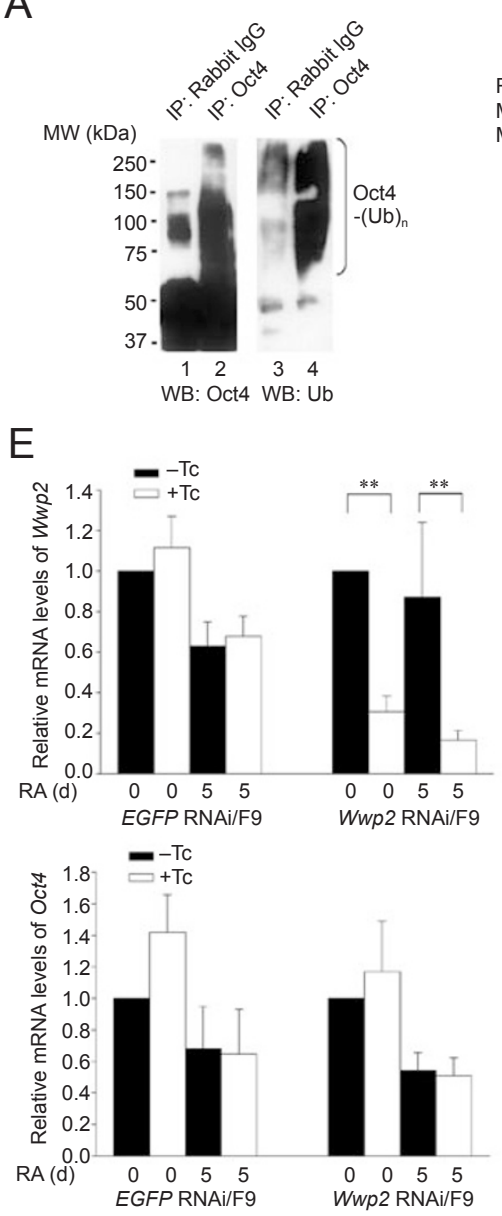

B

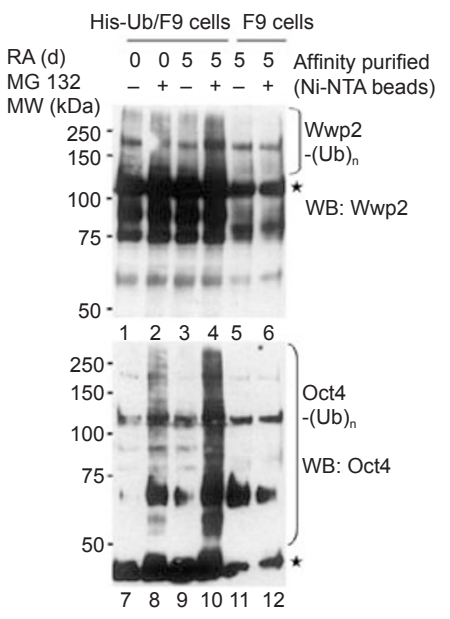

F

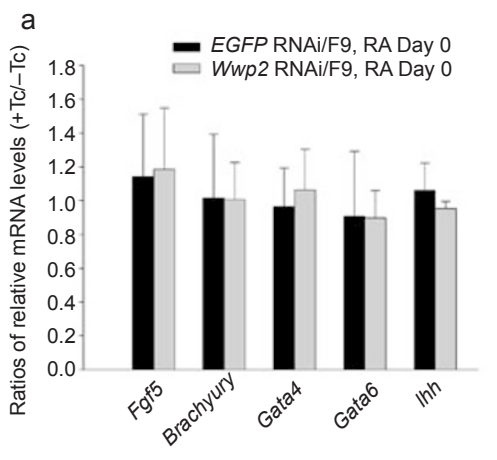

C
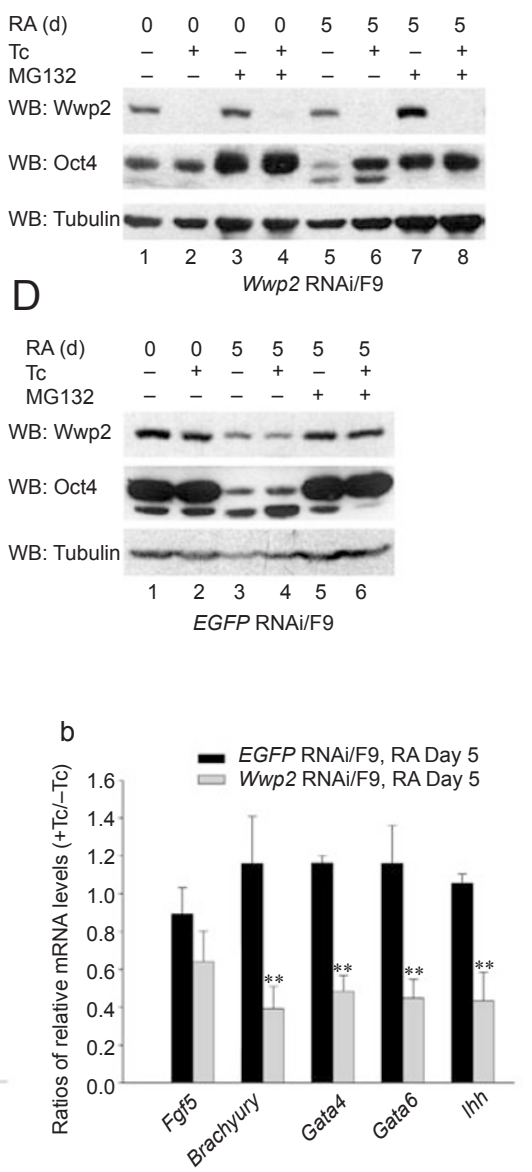

Figure 2 Wwp2 is an important regulator for Oct4 protein levels during differentiation processes. (A) Ubiquitination of endogenous Oct4 in CGR8 ESCs. After treating with MG132, cells were collected in RIPA buffer containing 1\% SDS, and cell extracts were subjected to immunoprecipitation assays with the Oct4 antibody or rabbit IgG. Subsequently, western blotting was performed to test ubiquitinated Oct4 with the Oct4 antibody (N124) and a Ub antibody, respectively. (B) Ubiquitination of Wwp2 and Oct4 increases along with the differentiation process. F9 cells expressing His-Ub (His-Ub/F9) and parental F9 cells were treated with or without RA for 5 days and collected after treatment with or without MG132. His-Ub tagged proteins in the lysates were isolated by affinity purification and analyzed by western blotting using antibodies against Wwp2 and Oct4 (N124), respectively. Asterisks, non-specific bands. (C) Wwp2 regulates Oct4 protein levels during RA-induced differentiation of F9 cells. F9 cells containing Wwp2 RNAi sequence (Wwp2 RNAi/F9) were treated with or without Tc for 3 days before they were induced by RA. The cell lysates were extracted after treatment with or without MG132 and analyzed by western blotting using the indicated antibodies. (D) The same experiments were performed in F9 cells containing EGFP RNAi sequence (EGFP RNAi/F9). Tubulin was utilized as loading control. (E) Down-regulation of Wwp2 does not affect the mRNA level of Oct4. The mRNA levels of Wwp2 and Oct4 were determined by qPCR. The level of mRNA in cells without RA and Tc treatment is defined as 1. (F) Knockdown of Wwp2 impairs the RA-induced differentiation process. The mRNA levels of differentiation markers were analyzed by qPCR. Relative mRNA level ratios of Tc treated versus untreated F9 cells were compared between EGFP RNAi/F9 and Wwp2 RNAi/F9 cells at an undifferentiated state (a) or a differentiated state (RA treatment for 5 days) (b). Data are shown as mean \pm SD ( $n=3$ experiments). $* * P<0.01$.

easy isolation of ubiquitinated proteins by nitrilotriacetic acid (NTA) affinity chromatography [33] and treated the cells with retinoid acid (RA) to induce differentiation, which is a well-characterized primitive endoderm differentiation model [37]. Western blot analysis of affinity- purified Ub-conjugates with a Wwp2 antibody showed that RA treatment for 5 days markedly enhanced Wwp2 ubiquitination (Figure 2B, compare lane 2 to lane 4). Notably, modification of Wwp2 accumulated with MG132 treatment (Figure 2B, compare lane 3 to lane 4), and 
the accumulation was more evident in cells treated with RA (Figure 2B, compare lane 3 to lane 4) than in cells without RA treatment (Figure 2B, compare lane 1 to lane $2)$. This phenomenon suggests that Ub-modification of Wwp2 and its degradation via the proteasome increased during RA-induced differentiation. Simultaneously, the level of ubiquitinated Oct4 increased significantly (Figure $2 \mathrm{~B}$, compare lane 8 to lane 10 ), and the ubiquitinated Oct4 proteins accumulated after MG132 treatment in both undifferentiated (Figure 2B, compare lanes 7 to lane 8 ) and differentiated F9 cells (compare lane 9 to lane 10). The enhanced Oct4 ubiquitination corresponded well to the higher level of Ub-modified Wwp2 during differentiation processes, implying a potential role of Wwp2 in controlling Oct4 protein levels during the RA-induced differentiation process.

To obtain direct evidence that Wwp2 regulates steadystate levels of Oct4 proteins during the differentiation process, we established a stable F9 cell line expressing Wwp2 RNAi that was induced upon addition of tetracycline (Tc). A stable F9 cell line expressing EGFP RNAi upon induction was also generated for Tc treatment control. As shown in Figure 2C (lane 2), Wwp2 protein levels became almost undetectable after treatment with Tc for three days. In agreement with the result that knockdown of $W w p 2$ does not affect the steady-state level of Oct4 proteins in undifferentiated CGR8 ESCs, down-regulation of $W w p 2$ did not appreciably alter Oct4 protein levels in undifferentiated F9 cells (Figure 2C, compare lane 1 to lane 2). However, Oct4 protein levels were obviously higher in the presence of MG132 (Figure 2C, compare lanes 1 and 2 to lanes 3 and 4), implying that other regulators, rather than Wwp2, might contribute to Oct4 protein degradation in undifferentiated F9 cells. As expected, Oct 4 protein levels significantly decreased at day 5 after RA treatment (compare lane 1 to lane 5). Notably, RA-induced reduction in the Oct4 protein level was abolished when $W w p 2$ expression was knocked down by Tc treatment (compare lane 5 to lane 6). Moreover, MG132 pretreatment masked the effect of $W w p 2$ RNAi on Oct4 protein levels (compare lane 7 to lane 8 ). These results provide evidence for Wwp2 acting as an important Ub E3 ligase mediating RA-induced Oct4 degradation through the $26 \mathrm{~S}$ proteasome. As negative control, Tc treatment did not interfere with RA-induced decrease in Oct4 protein levels in F9 cells expressing EGFP RNAi (Figure 2D), indicating that it was downregulation of $W w p 2$ expression but not Tc treatment that eliminated RA-induced Oct4 protein degradation. Therefore, Wwp2 participates in the precise control of endogenous Oct4 protein levels during RA-induced differentiation of F9 cells.
To exclude the possibility that the elevated Oct4 protein level observed when $W w p 2$ was knocked down was brought about at a transcriptional level, we evaluated Oct 4 mRNA levels by quantitative RT-PCR (qPCR) analysis. Figure 2E showed that $\mathrm{Tc}$ treatment reduced the Wwp 2 transcript level upto $20 \%-30 \%$ compared to that of untreated cells. However, the Oct 4 mRNA level was not affected by Tc treatment regardless of the presence or absence of RA. Moreover, the finding that MG132 treatment not only blocked Oct 4 protein degradation but also eliminated influence of $W w p 2$ RNAi on Oct4 protein levels (Figure 2C, lanes 7 and 8 ) is indicative of an effect of $W w p 2$ RNAi on the Oct4 level at a post-translational level.

As a precisely controlled dosage of Oct4 is required to sustain self-renewal and determine distinct differentiation processes in pluripotent stem cells [4], we were curious about whether $W w p 2$ RNAi-mediated interference with Oct4 protein degradation would disturb normal RAinduced differentiation processes. Therefore, we compared the gene expression patterns of known embryonic germ layer markers between RA-induced F9 cells with and without Tc treatment. qPCR analysis revealed that RA treatment dramatically elevated expression of endoderm markers such as Gata4, Gata6 and Ihh, and slightly enhanced expression of the primitive ectoderm marker Fgf5 as well as the mesoderm marker brachyury, indicating efficient induction of primitive endoderm differentiation by RA (Supplementary information, Figure S1). Importantly, down-regulation of $W w p 2$ expression siginificantly reduced RA-induced activation of these germ layer markers, including brachyury, Gata4, Gata6 and Ihh (Figure 2F, b). In contrast, knockdown of $W w p 2$ did not affect the expression of these markers in undifferentiated F9 cells (Figure 2F, a). These results strongly argue that Wwp2 is an important regulator for an appropriate differentiation process to occur in ECCs and it may function through a dynamic control of Oct4 protein levels.

\section{Wwp2 regulates Oct4 ubiquitination and degradation in a dosage-dependent manner}

Data presented above demonstrated a specific role of Wwp2 in Oct4 degradation during differentiation processes. However, expression of Wwp2 decreased along with the differentiation process (Figures 2C-2E). It appears that Wwp2 does not affect Oct4 protein levels when its expression level is high in undifferentiated cells, and that the reduction in $W w p 2$ expression levels during differentiation may activate its function. Therefore, we hypothesized that Wwp2 promotes Oct4 ubiquitination in a dosage-dependent manner. To test this hypothesis, we performed an in vitro ubiquitination assay in the pres- 
ence of a constant amount of GST-Oct4 and increasing amounts of GST-Wwp2 or GST-Itch. The latter is also a Ub E3 ligase of Oct4 (Bing Liao and Ying Jin, unpublished data) and is used here as control. Interestingly, strong ubiquitination signals were observed when GSTWwp2 was at low dosages $(0.2$ and $0.4 \mu \mathrm{g})$, whereas the signals became weaker when dosages of Wwp2 were higher (Figure 3A, lanes 1-4). By contrast, GST-Itch catalyzed ubiquitination at a constant efficiency regardless of its dosage (Figure 3A, lanes 5-8), implying that the dosage-dependency is specific to Wwp2. Of note, the ubiquitination signals detected by the Ub antibody in such analysis could also result from Wwp2 auto-ubiquitination. To exclude this possibility, immunoprecipitation assays with the Oct4 antibody were performed prior to western blot analysis. As shown in Figure 3B, the extent of ubiquitinated Oct4 signals became weaker as more Wwp2 proteins were added in the reactions, verifying that Wwp2 indeed regulates Oct4 ubiquitination in a dosage-dependent manner.

We next tested whether the enzymatic activity of Wwp2 was suppressed by high levels of Wwp2 in vivo.
Proteins modified by Ub in HEK 293 cells were purified by Ni-NTA beads and analyzed by western blotting using the Oct4 antibody. As shown in Figure 3C (top panel), a small increase in the Wwp2 dosage ( 1 and $2 \mu \mathrm{g})$ enhanced Oct4 ubiquitination. However, further increase in Wwp2 dosages ( 4 and $8 \mu \mathrm{g}$ ) reduced ubiquitination of Oct4. Hence, higher the levels of Wwp2 dosages were, fewer the amount of Oct4 proteins that was ubiquitinated (lanes 1-10). This is consistent with our in vitro observation. In parallel with the ubiquitination pattern, Oct4 proteins in whole cell lysates were at lower levels in cells expressing low dosages of Wwp2 (Figure 3C, bottom panel, compare lane 1 to lanes 3 and 5), but became more stable when the Wwp2 dosage increased (lanes 7 and 9). Moreover, we compared levels of exogenously expressed Wwp2 in HEK 293 cells and that of endogenous Wwp2 proteins in F9 cells, and found that the protein level of exogenous Wwp2 produced by $8 \mu \mathrm{g}$ of Wwp2 expression vectors was similar to that of Wwp2 proteins in undifferentiated F9 cells (Supplementary information, Figure S2). Under both conditions, Wwp2 showed suppressed enzymatic activity in Oct4 ubiquitination. Taken
A

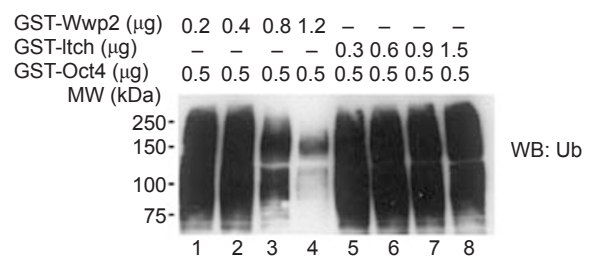

B

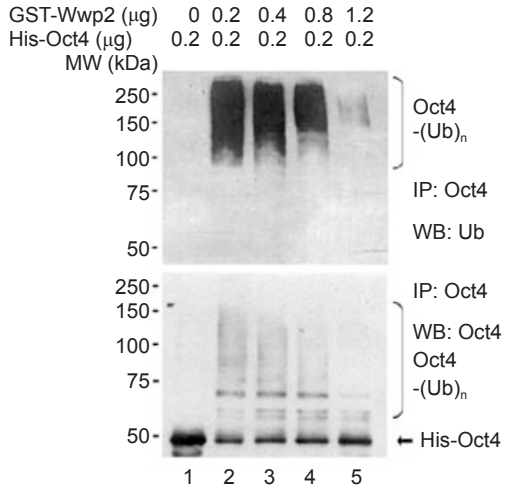

C

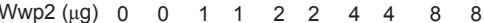

$\begin{array}{lllllllllll}\text { Oct4 }(\mu \mathrm{g}) & 2 & 2 & 2 & 2 & 2 & 2 & 2 & 2 & 2 & 2\end{array}$

MG132 - + - + $-+{ }^{+}+\ldots+\ldots+$

$\mathrm{MW}(\mathrm{kDa}) \quad$ Affinity purified

250 - $\quad$ (Ni-NTA Beads)

150. $\quad$ WB: Oct4

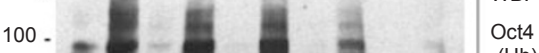

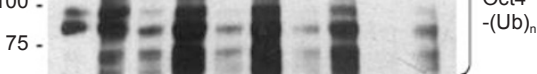

50

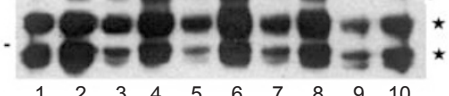

$\begin{array}{llllllllll}1 & 2 & 3 & 4 & 5 & 6 & 7 & 8 & 9 & 10\end{array}$

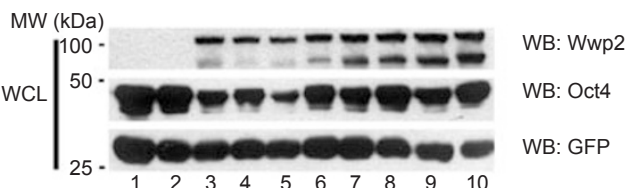

Figure 3 Wwp2 targets Oct4 for ubiquitination and degradation in a dosage-dependent manner. (A) In vitro ubiquitination mediated by various amounts of GST-Wwp2 (lanes 1-4) or GST-Itch (lanes 5-8) was examined by western blotting using a Ub antibody. E1, E2 and His-Ub were included in all reactions. (B) In vitro ubiquitination of His-Oct4 (0.2 $\mu \mathrm{g})$ by various amounts of GST-Wwp2. After immunoprecipitation of reaction products with Oct4 antibody (N124), the ubiquitinated Oct4 were tested by western blotting with a Ub antibody (P4D1) and Oct4 antibody (N124), respectively. (C) Cell lysates from HEK 293 cells expressing His-Ub $(4 \mu \mathrm{g})$, GFP $(2 \mu \mathrm{g})$, Oct4 $(2 \mu \mathrm{g})$ and Wwp2 $(0,1,2,4$ or $8 \mu \mathrm{g})$ were purified by Ni-NTA affinity-beads and analyzed by western blotting with Oct4 antibody (N124). The cells were treated with or without MG132 before harvest. In the bottom panel, the whole cell lysates (WCL) were subjected to western blotting using antibodies against Wwp2, Oct4 and GFP, respectively. GFP served as loading control. Asterisks, non-specific bands. 
together, the above data indicate that Wwp2 targets Oct4 for ubiquitination and degradation in a dosage-dependent manner, and that differentiation-related reduction in the Wwp2 expression level might provide an optimal concentration for Wwp2 to modify Oct4 post-translationally.

Auto-ubiquitination of Wwp2 occurs through an intramolecular mechanism in a dosage-dependent manner

To characterize the inhibitory effect of Wwp2 on Oct4 ubiquitination observed at its higher dosages, we examined whether the higher dosages of Wwp2 would affect its own ubiquitination, as the extent of auto-ubiquitination of E3 ligases often corresponds to their own enzymatic activity $[31,38]$. The result from our in vitro auto-ubiquitination assays showed that the level of ubiquitinated Wwp2 was highest when GST-Wwp2 was at a dosage of $0.3 \mu \mathrm{g}$ (Figure 4A, lane 2). Further increase in the GST-Wwp2 dosage to $1.0 \mu \mathrm{g}$ decreased the level of auto-ubiquitinated Wwp2 substantially (lane 3). In contrast, auto-ubiquitination of Itch increased gradually when the amount of GST-Itch was elevated from 0.3 to $1.0 \mu \mathrm{g}$ (lanes 4-6). This result suggests that the inhibitory effect of Wwp2 at higher dosages on Oct4 ubiquitination is probably caused by suppression of its own enzymatic activity and is a specific property of Wwp2. In another word, this phenomenon occurs irrespective of the presence of its substrate.

To elucidate the molecular mechanisms underlying Wwp2 auto-modification, we studied whether Wwp2 auto-ubiquitination occurs through an inter-molecular or intra-molecular mechanism. To this end, both WT GSTWwp2 and a mutant Wwp2 (Wwp2-CA), in which an essential cysteine residue for its ligase activity was mutated to alanine residue, were used as substrates of WT ligase His-Wwp2 in an in vitro ubiquitination assay. As shown in Figure 4B, GST-Wwp2-CA cannot be ubiquitinated by His-Wwp2 (lanes 3-5), implicating that Wwp2 cannot catalyze the transfer of $\mathrm{Ub}$ molecules from its cysteine to another Wwp2 (Wwp2-CA). However, unlike GSTWwp2-CA, auto-ubiquitination of WT GST-Wwp2 was easily detected (lanes 7-10), suggesting that auto-ubiquitination of Wwp2 probably occurs through an intramolecular mechanism.

As shown in Figures 1A and 1B, Wwp2 catalyzes Lys 63-linked poly-Ub chain formation on Oct4. We predicted that auto-ubiquitination of Wwp2 could also involve the Lys 63-linkage. As predicted and shown in the Supplementary information, Figure S3A and S3B, auto-ubiquitination of Wwp2 was also formed through Lys 63-linked poly-Ub chains, similar to that of Wwp2mediated Oct4 poly-ubiquitination.

Wwp2 dimerizes in vitro and in vivo, interfering with its ligase activity

Based on the above findings, we hypothesized that homodimerization of Wwp2 might explain the suppression of the ligase activity at higher dosages. To test this, we first determined whether dimerization occurred between

A

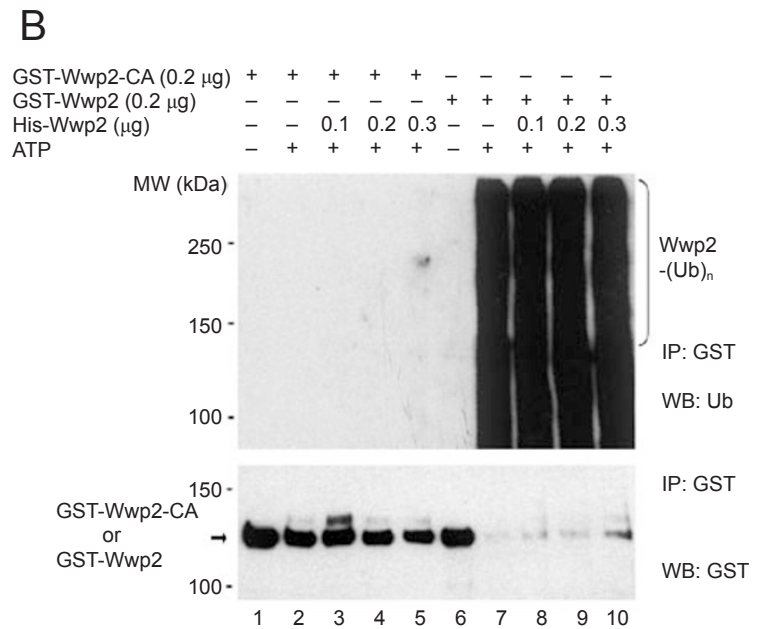

Figure 4 Auto-ubiquitination of Wwp2 occurs through an intra-molecular mechanism dosage-dependently. (A) Auto-ubiquitination of Wwp2 and Itch at different dosages. Various amounts of GST-Wwp2 or GST-Itch $(0.1,0.3,1.0 \mu \mathrm{g})$ were added to in vitro ubiquitination reactions, and samples were analyzed by western blotting with a Ub antibody. (B) The Wwp2-CA mutant cannot generate auto-ubiquitination. The in vitro ubiquitination assays were performed using GST-Wwp2-CA (lanes 1-5) or GST-Wwp2 (lanes 6-10) as a substrate for His-Wwp2. After immunoprecipitation of reaction products with GST antibody, the western blot was analyzed using a Ub antibody (top panel), and reprobed by a GST antibody (bottom panel). 
Wwp2 molecules. The result from GST pull-down assays shows that, in addition to GST-tagged full-length Wwp2, GST fusion proteins of truncated C2, WW (including all four WW domains), HECT domains were all capable of binding to His-tagged Wwp2, as revealed by western blot analysis using anti-His antibody (Figure 5A, top panel). The finding indicates that Wwp2 homodimers can be generated in vitro, and that all three domains of Wwp2 are able to associate with full-length Wwp2. Subsequently, we determined whether Wwp2 associated with each other in vivo. Coimmunoprecipitation (CoIP) assays revealed that GFP-tagged Wwp2 coimmunoprecipitated with FLAG-tagged Wwp2 (Figure 5B, the first row, lanes 3 and 4), providing evidence for the formation of Wwp2 homodimers in mammalian cells.

To further verify that Wwp2 dimerization can influence its own ligase activity, varying amounts of GST-C2 fusion proteins were incubated with GST-Wwp2 in an in vitro ubiquitination assay. Similar to full-length Wwp2, higher levels of GST-C2 proteins repressed the autoubiquitination of Wwp2 in vitro in a dosage-dependent fashion, as revealed by antibodies against $\mathrm{Ub}$ and $\mathrm{Wwp} 2$, respectively (Figure 5C). The suppression of $\mathrm{Wwp} 2$ auto-ubiquitination could be caused by the increased direct interaction between the full-length $\mathrm{Wwp} 2$ and $\mathrm{C} 2$ domain at higher dosages. Therefore, Wwp2 molecules could form homodimers both in vitro and in vivo, which might explain why Wwp2 represses its own Ub ligase activity when it is present at higher dosages.

The N-terminus of Wwp2 is essential for its ligase activity

It was reported that interaction between $\mathrm{C} 2$ and HECT domains of the C2-WW-HECT E3 ligase Smurf2 autoinhibits its ligase activity to protect itself and its substrates from futile degradation in cells [38]. We were interested in knowing whether Wwp2 would have a similar property. Thus, we compared the ligase activity between
A

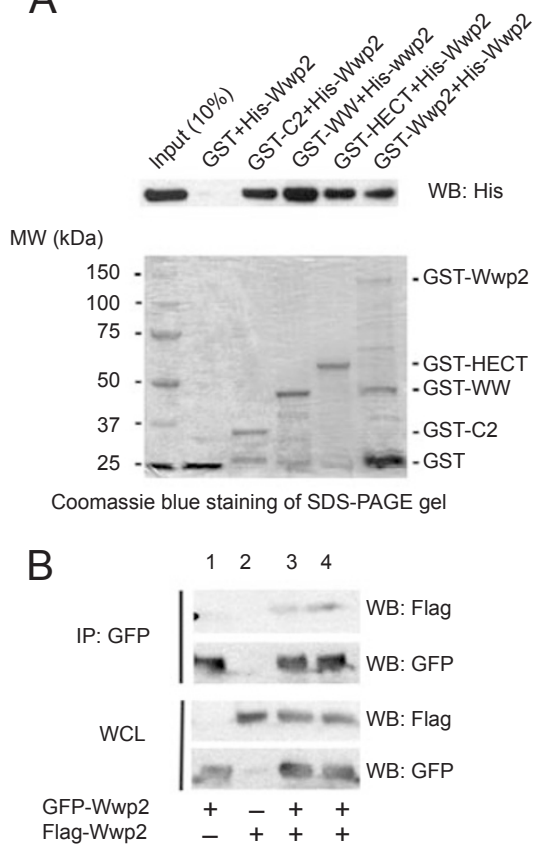

C

GST-Wwp2 + + + + +

ATP ++++

GST-C2 $(\mu \mathrm{g})-0.250 .5 \quad 1.01 .0$

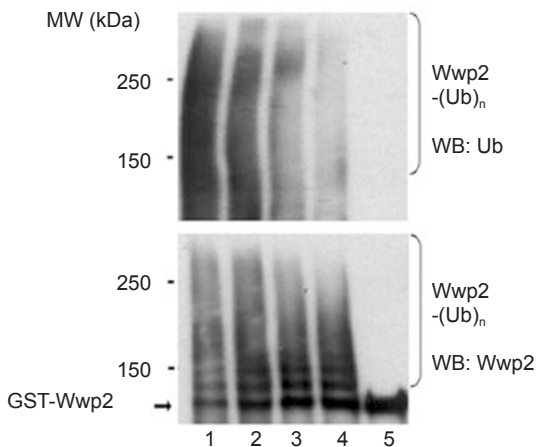

Figure 5 Homodimierization of Wwp2 interferes with its ligase activity. (A) GST fusion proteins of C2, WW, HECT domains and WT Wwp2 bind to His-Wwp2. Fractions from GST pull-down assays were analyzed by western blotting with a His antibody (top panel). GST and GST fusion proteins of Wwp2 were separated on SDS-PAGE gel and stained by Coomassie Blue (bottom panel). (B) ColP assays of Flag-Wwp2 and GFP-Wwp2 in COS7 cells. Expression vectors encoding Flag-Wwp2 and GFP-Wwp2 were cotransfected into COS7 cells. Whole cell lysates (WCL) were immunoprecipitated (IP) with a GFP antibody and analyzed by western blotting with a Flag antibody or GFP antibody (top panel). The expression levels of FlagWwp2 and GFP-Wwp2 in COS7 cells were examined by western blotting with antibodies against Flag and GFP, respectively. Lanes 3 and 4 are duplicated samples. (C) Auto-ubiquitination of GST-Wwp2 $(0.2 \mu \mathrm{g})$ was examined in the presence of various amounts of GST-C2 in an in vitro ubiquitination assay. Western blot analysis was performed with Ub antibody or Wwp2 antibody, respectively. E1, E2 and wild-type Ub were present in all reactions. 
GST-fusion proteins of full-length Wwp2 and truncated Wwp2 containing only WW and HECT domains (amino acids 302-870) in in vitro ubiquitination assays. Surprisingly, deletion of $\mathrm{N}$-terminal 301 amino acid residues from Wwp2 abrogated its ability to ubiquitinate Oct4 completely, while full-length Wwp2 catalyzed Oct4 poly-ubiquitination efficiently under the same experimental conditions (Figure 6, top panel). Consistent with this result, the unmodified form of Oct 4 proteins diminished dramatically in the reaction containing full-length Wwp2, whereas it was barely changed in the reactions containing the truncated Wwp2. Similarly, auto-polyubiquitination of Wwp2 was only detected in the presence of the full-length Wwp2 and not in the presence of the truncated Wwp2 (Figure 6, middle panel). Expression of GST-fusion proteins of the full-length and truncated Wwp2 was confirmed by western blot analysis (Figure 6 , bottom panel). Of note, a single $\mathrm{Ub}$ attached to the truncated Wwp2 was detected using the GST antibody (lanes 3 and 4). Failure to detect the Ub attachment to the truncated Wwp2 by the Ub antibody (the middle panel) could be due to its relatively low affinity to the mono-Ub modification. These observations indicate that the $\mathrm{N}$-terminus is required for the ligase activity of Wwp2 and that the ability of Wwp2 to ubiquitinate Oct4 might be dependent on its auto-ubiquitination.

\section{Discussion}

Oct4 is an essential factor for ESCs to maintain selfrenewal and pluripotency as well as for reprogramming somatic cells into pluripotency. Strict dependence of its functions on the intracellular dosage is a unique characteristic of Oct4. Therefore, understanding molecular mechanisms by which the Oct4 dosage is dynamically controlled is fundamental for stem cell biology. Although transcriptional regulation is believed to be primarily responsible for down-regulation of Oct 4 expression during ESC differentiation, a previous study reported that the protein level of Oct4 is significantly down-regulated, while the Oct4 transcript is not changed during the process of primitive endoderm differentiation [36]. Results from the current study further demonstrate that posttranslational modification can serve as an important complementary mechanism to transcriptional regulation of Oct 4 in a timely and selective manner during differentiation of ECCs.

It is generally accepted that different Ub linkages could lead to various conformations of Ub chains and generate diverse molecular signals in cells. Using a ubiquitination system developed by Richard Baer [39] to ensure the proper fidelity of linkage formation in vitro, we

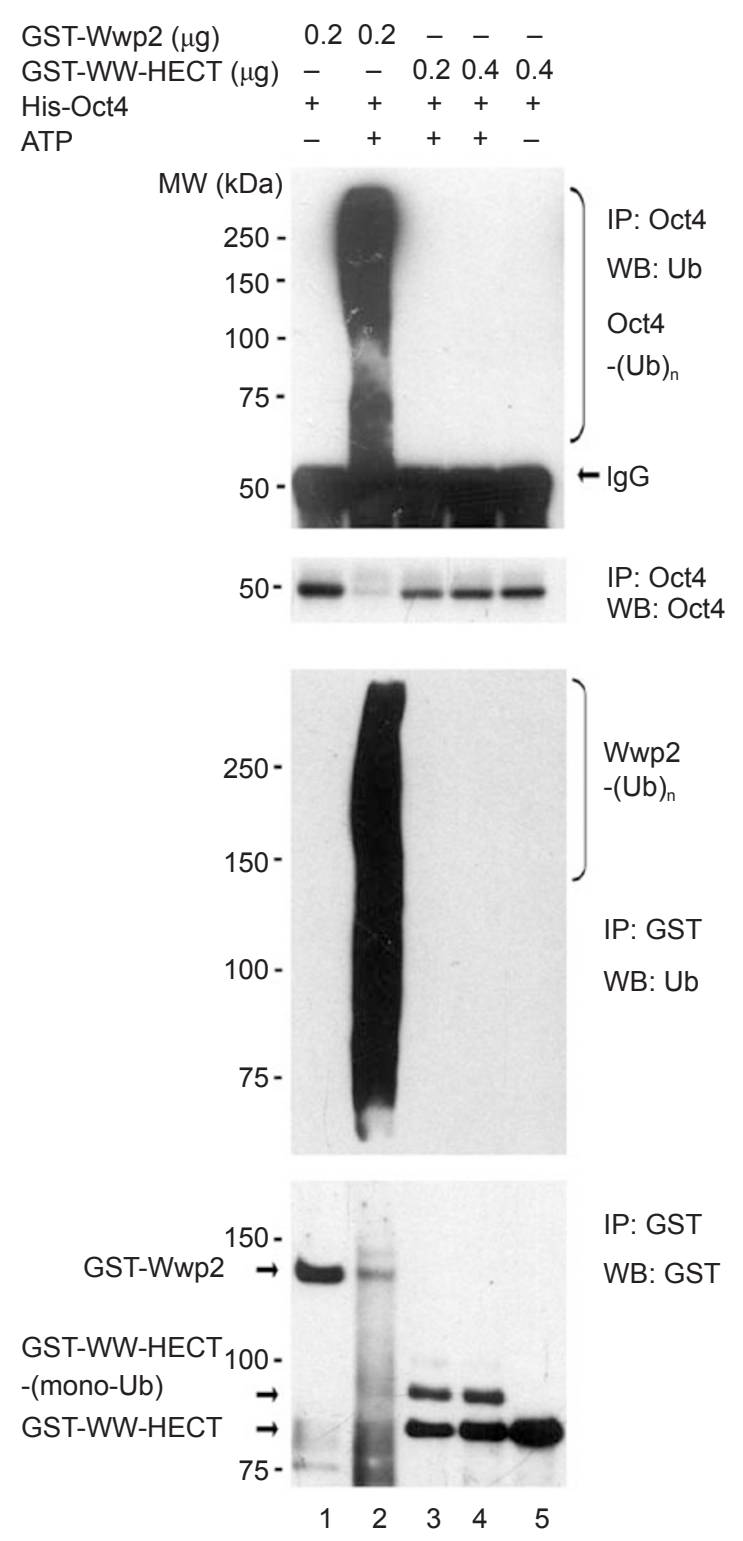

Figure 6 The N-terminus of Wwp2 is essential for its ligase activity. GST fusion proteins of full-length Wwp2 and WW-HECTdomain were used for in vitro ubiquitination assays in the presence or absence ATP. E1, E2, Ub and His-Oct4 were included in all reactions. The western blot was analyzed with a Ub antibody to detect ubiquitinated Oct4 and auto-ubiquitinated Wwp2 after immunoprecipitation, and reprobed with antibodies against Oct4 (N124) and GST to detect His-oct4, GST-Wwp2 and GST-WWHECT, respectively.

clearly demonstrate that interaction between Wwp2 and Oct4 generates chains composed of a single kind of the Lys 63 linkage. Recently, Saeki et al. showed that Lys 63-linked poly-Ub chains may serve as a targeting signal for the $26 \mathrm{~S}$ proteasome in yeast cells [40]. However, 
evidence for such a reaction occurring in mammalian cells is lacking. Data from both our previous and current studies indicate that Wwp2-mediated Lys 63-linked poly-ubiquitination promotes degradation of ectopically as well as endogenously expressed Oct4 through the Ub-proteasome pathway, implying association of Lys 63 -linked poly-Ub chains with the proteolytic pathway in mammalian cells.

Ub E3 ligases are finely controlled by a variety of mechanisms [38, 41-44]. Among multiple types of regulation, auto-ubiquitination is known as a means for cells to control the abundance of Ub E3 ligases [38]. However, recent studies have implicated that the auto-ubiquitination activity of certain Ub E3 ligases is closely related to their catalytic capacity and is required for mediating their downstream signaling [31]. For instance, auto-ubiquitination of Nedd4, a Rsp5-family member, plays an essential role in determining its ability to modify the substrates [45]. Our finding that Wwp2 loses the ability to ubiquitinate Oct4 when the auto-ubiquitination is eliminated by deletion of its N-terminus suggests that autoubiquitination might be essential for the ligase activity of Wwp2. The dependence on its N-terminus for Wwp2 auto-ubiquitination could be explained by two possibilities: one is that the absence of $\mathrm{N}$-terminus disrupts the normal configuration required for the Wwp2 enzymatic activity and the other one is that the $\mathrm{N}$-terminus contains critical Lys residues accepting $\mathrm{Ub}$ during its auto-ubiquitination. In case of Smurf2, the C2 domain interacts with the HECT domain to inhibit its ligase activity through an intra-molecular mechanism [38]. Thus, Wwp2 and Smurf2 modulate their own catalytic activity through different manners, even though they are both C2-WW-HECTtype E3 ligases. Nevertheless, for both enzymes, autoubiquitination is important to modify their substrates. Interestingly, Wiesner et al. [38] found that, like Smurf2, deletion of the C2 domain of human WWP2 significantly enhanced its auto-ubiquitination, suggesting that human WWP2 may behave differently from its murine counterpart. Recently, we reported that human WWP2 [46] also targets human OCT4 for ubiquitination and degradation through the $26 \mathrm{~S}$ proteasome. Unlike Wwp2, WWP2 promotes OCT4 degradation in undifferentiated human ESCs and does not display an inhibition phenomenon at higher dosages. This is consistent with the current view that differences exist between human and mouse ESCs, although they are similar in many ways [47].

Uniquely, auto-ubiquitination of Wwp2 depends on its own dosages. This property has not been reported for other Ub E3 ligases. Instead of inhibition of the catalytic activity, RING-type E3 ligase Mdm2 switches the Ub modification of $\mathrm{p} 53$ from mono- to poly- ubiquitination at higher concentrations [48], suggesting that dosagedependent regulation is one of the mechanisms to control Ub E3 ligases. Based on our findings that Wwp2 can dimerize both in vitro and in vivo and that increasing levels of the $\mathrm{C} 2$ domain result in suppression of Wwp2 autoubiquitination through an inter-molecular mechanism, we propose that Wwp2 homodimerization may be, at least partially, responsible for the inhibitory effect of higher levels of Wwp2 on its own catalytic activity. It is possible that Wwp2 dimerization covers the ligase activity site or induces conformational changes to inhibit its ligase activity. Altogether, these data unveil a novel phenomenon and potential mechanism for Wwp2 to modulate its own enzymatic activity, allowing a further in-depth dissection of multiple layers of E3 ligase regulation.

Data from this study suggest that the level of Wwp2 in undifferentiated ESCs or ECCs might be higher than its optimal level for Oct4 ubiquitination and degradation. We suspect that the rationality for such a high level of Wwp2 may come from the need of its other substrates co-expressed in undifferentiated ESCs and ECCs. Indeed, we have previously demonstrated that Rpb1, the largest subunit of the RNA polymerase II, is also a substrate of Wwp2 and that Wwp2 is required for maintenance of Rpb1 at an appropriate level in undifferentiated ECCs [49]. It is worthy to note that Wwp2-mediated Rpb1 degradation is not inhibited by its higher dosages (data not shown). Therefore, the differential optimal dosage for an E3 ligase could serve to discriminate its distinct substrates even when they are distributed in the same cellular compartment at the same time. However, it is also possible that other factors are also needed in this differential regulation, as mere reduction of the Wwp2 level by its specific RNAi in undifferentiated ESCs and ECCs could not engage it in the regulation of Oct4 protein levels, suggesting that other differentiation-regulated factors may also associate with Wwp2-mediated Oct4 ubiquitination and degradation during differentiation processes. In addition, Wwp2 at higher concentrations might compete away other more efficient Ub E3 ligases present in undifferentiated ESCs or ECCs. Further investigation is required to elucidate the physiological significance and molecular mechanism underlying the unique feature of Wwp2.

In conclusion, our data demonstrate that Wwp2-mediated Oct4 poly-ubiquitination and its auto-ubiquitination occur through the Lys 63 linkage and may be involved in down-regulation of Oct4 protein levels. Moreover, we present a new dosage-dependent mechanism by which the Ub E3 ligase may modulate its own activity and discriminate its distinct substrates under specific cellular contexts. Importantly, we provide evidence for an impor- 
tant role of Wwp2-mediated Oct4 ubiquitination during differentiation processes. The findings will help us to understand how Oct4 protein levels are dynamically controlled in ESCs. On the other hand, Ub E3 ligases have attracted considerable attention because their dysfunction has been implicated in embryonic development defects and cancer or disease progression. The results obtained in this study will open up new avenues for designing or screening agents that can modulate activities of diseaseassociated ligases.

\section{Materials and Methods}

\section{Plasmid construction}

Plasmids utilized for expression of Oct4, Wwp2, Wwp2-CA mutant or individual Wwp2 domains have been described previously [33, 49]. His-tagged Ub K0 mutant, un-tagged WT and mutant $\mathrm{Ub}$ expression vectors were kindly provided by Dr Richard Baer. His-tagged Ub expression vector pMT107 was a gift from Dr Dirk Bohmann.

\section{Reagents and antibodies}

The E1, E2, recombinant His-tagged WT Ub and pure 26S proteasomes were purchased from Calbiochem. MG132, G418, Tc and RA were from Sigma. Antibodies used include: Oct4 (C10, Santa Cruz), Tubulin (Sigma), GFP (Roche), Flag (Sigma), His (Sigma) and Ub (P4D1, Cell Signaling). Antibodies to Oct4 (N124 and C70), Wwp2 and GST were generated as described previously [33].

\section{Cell culture and transfection}

HEK 293 cells and COS7 cells were maintained under standard conditions, and transfected using the standard calcium phosphate precipitation or Lipofectamine 2000 (Invitrogen), respectively. F9 ECCs were cultured as suggested by ATCC. For the stable cell line, the His-Ub expression vector was introduced into F9 cells and selected with $600 \mu \mathrm{g} / \mathrm{ml} \mathrm{G} 418$. The mouse CGR8 ESC line was kindly provided by Dr Austin Smith and cultured as described previously [50]. The stable cell lines for Tc induced RNAi were generated as described previously [49].

\section{$q P C R$ analysis}

Total RNA was extracted using TRIzol reagent (Invitrogen) and reverse-transcribed into cDNA using oligo $(\mathrm{dT})_{15}$ and ReverTra Ace reverse transcriptase (TOYOBO). qPCR analysis was carried out as previously described [51]. The sequences of primers are provided in Supplementary information, Table S1.

\section{RA-induced differentiation of F9 cells}

To induce differentiation of F9 cells toward primitive endoderm, cells were plated at a density of 40000 cells per 6 -cm dish coated with gelatin. RA at $50 \mathrm{nM}$ was added $24 \mathrm{~h}$ after cell plating. Cells were collected for RT-PCR and western blot analysis after $5 \mathrm{~d}$.

\section{Western blot analysis}

Protein concentrations were determined using the BCA protein assay kit (Pierce). For experiments involving transient transfection, the cotransfected pSV- $\beta$-galactosidase plasmid (a kind gift from R Baer) was utilized to normalize each sample. Detection was performed with enhanced chemiluminescence (Pierce). All experiments were repeated three times and representative data are shown.

\section{Expression and purification of fusion proteins}

GST and His fusion proteins were expressed and purified according to the manufacturers instructions from Amersham Biosciences and Novagen, respectively. Un-tagged WT and mutant Ubs were expressed in Rosetta (DE3) pLysS cells (Novagen) and purified as described previously [39].

\section{GST pull-down and immunoprecipitation assays}

GST fusion proteins $(0.5 \mu \mathrm{g})$ bound with glutathione-sepharose 4B beads were incubated with His fusion proteins $(1.0 \mu \mathrm{g})$ in $500 \mu \mathrm{l}$ of the TBS-N buffer (20 mM Tris- $\mathrm{HCl}, \mathrm{pH} 7.6,300 \mathrm{mM} \mathrm{NaCl}$ and $0.1 \% \mathrm{NP}-40$ ) at $4{ }^{\circ} \mathrm{C}$ for $4 \mathrm{~h}$. For CoIP experiments, cell lysates were prepared in the CoIP buffer [49] and incubated with a specific antibody for $4 \mathrm{~h}$ at $4{ }^{\circ} \mathrm{C}$, followed by addition of protein ASepharose beads for another $2 \mathrm{~h}$. The samples from these assays were analyzed by western blotting.

\section{Ubiquitination assay in vitro and in vivo}

In vitro and in vivo ubiquitination assays were performed as described previously [33, 49].

\section{Acknowledgments}

We thank Dr Richard Baer (Pathology, Columbia University, New York, USA) for generously providing various Ub mutant plasmids. This study was supported by Grants from the National Natural Science Foundation of China (30871257, 30730051) and the National High Technology Research, Development Program of China (2006CB943901 and 2007CB947904), the Shanghai Science and Technology Developmental Foundation (08JC1413100) and the Shanghai Leading Academic Discipline Project (S30201).

\section{References}

1 Scholer HR, Ruppert S, Suzuki N, Chowdhury K, Gruss P. New type of POU domain in germ line-specific protein Oct-4. Nature 1990; 344:435-439.

2 Palmieri SL, Peter W, Hess H, Scholer HR. Oct-4 transcription factor is differentially expressed in the mouse embryo during establishment of the first two extraembryonic cell lineages involved in implantation. Dev Biol 1994; 166:259-267.

3 Schoorlemmer J, Jonk L, Sanbing S, et al. Regulation of Oct4 gene expression during differentiation of EC cells. Mol Biol Rep 1995; 21:129-140.

4 Niwa H, Miyazaki J, Smith AG. Quantitative expression of Oct-3/4 defines differentiation, dedifferentiation or selfrenewal of ES cells. Nat Genet 2000; 24:372-376.

5 Takahashi K, Yamanaka S. Induction of pluripotent stem cells from mouse embryonic and adult fibroblast cultures by defined factors. Cell 2006; 126:663-676.

6 Kim JB, Sebastiano V, Wu G, et al. Oct4-induced pluripotency in adult neural stem cells. Cell 2009; 136:411-419.

7 Adhikary S, Marinoni F, Hock A, et al. The ubiquitin ligase HectH9 regulates transcriptional activation by Myc and is es- 
sential for tumor cell proliferation. Cell 2005; 123:409-421.

8 Alexandru G, Graumann J, Smith GT, et al. UBXD7 binds multiple ubiquitin ligases and implicates p97 in HIF1alpha turnover. Cell 2008; 134:804-816.

9 Zhong Q, Gao W, Du F, Wang X. Mule/ARF-BP1, a BH3only E3 ubiquitin ligase, catalyzes the polyubiquitination of Mcl-1 and regulates apoptosis. Cell 2005; 121:1085-1095.

10 Melino G, Gallagher E, Aqeilan RI, et al. Itch: a HECT-type E3 ligase regulating immunity, skin and cancer. Cell Death Differ 2008; 15:1103-1112.

11 Dunn R, Klos DA, Adler AS, Hicke L. The C2 domain of the Rsp5 ubiquitin ligase binds membrane phosphoinositides and directs ubiquitination of endosomal cargo. J Cell Biol 2004; 165:135-144.

12 Huibregtse JM, Scheffner M, Beaudenon S, Howley PM. A family of proteins structurally and functionally related to the E6-AP ubiquitin-protein ligase. Proc Natl Acad Sci USA 1995; 92:2563-2567.

13 Chen HI, Sudol M. The WW domain of Yes-associated protein binds a proline-rich ligand that differs from the consensus established for Src homology 3-binding modules. Proc Natl Acad Sci USA 1995; 92:7819-7823.

14 Jennings MD, Blankley RT, Baron M, Golovanov AP, Avis JM. Specificity and autoregulation of Notch binding by tandem WW domains in suppressor of Deltex. J Biol Chem 2007; 282:29032-29042.

15 Sudol M, Recinos CC, Abraczinskas J, Humbert J, Farooq A. WW or WoW: the WW domains in a union of bliss. IUBMB Life 2005; 57:773-778.

16 Ingham RJ, Colwill K, Howard C, et al. WW domains provide a platform for the assembly of multiprotein networks. Mol Cell Biol 2005; 25:7092-7106.

17 Hershko A, Heller H, Elias S, Ciechanover A. Components of ubiquitin-protein ligase system. Resolution, affinity purification, and role in protein breakdown. J Biol Chem 1983; 258:8206-8214.

18 Ciechanover A. The ubiquitin-proteasome proteolytic pathway. Cell 1994; 79:13-21.

19 Hershko A. The ubiquitin system for protein degradation and some of its roles in the control of the cell division cycle. Cell Death Differ 2005; 12:1191-1197.

20 Hershko A, Ciechanover A. The ubiquitin system. Annu Rev Biochem 1998; 67:425-479.

21 Kim HT, Kim KP, Lledias F, et al. Certain pairs of ubiquitinconjugating enzymes (E2s) and ubiquitin-protein ligases (E3s) synthesize nondegradable forked ubiquitin chains containing all possible isopeptide linkages. J Biol Chem 2007; 282:17375-17386.

22 Hadari T, Warms JV, Rose IA, Hershko A. A ubiquitin Cterminal isopeptidase that acts on polyubiquitin chains. Role in protein degradation. J Biol Chem 1992; 267:719-727.

23 Brusky J, Zhu Y, Xiao W. UBC13, a DNA-damage-inducible gene, is a member of the error-free postreplication repair pathway in Saccharomyces cerevisiae. Curr Genet 2000; 37:168174.

24 Wu CJ, Conze DB, Li T, Srinivasula SM, Ashwell JD. Sensing of Lys 63-linked polyubiquitination by NEMO is a key

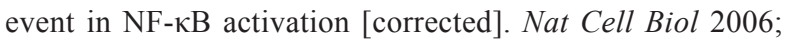
8:398-406.
25 Geetha T, Jiang J, Wooten MW. Lysine 63 polyubiquitination of the nerve growth factor receptor TrkA directs internalization and signaling. Mol Cell 2005; 20:301-312.

26 Shekhar MP, Gerard B, Pauley RJ, Williams BO, Tait L. Rad6B is a positive regulator of $\beta$-catenin stabilization. Cancer Res 2008; 68:1741-1750.

27 Hofmann RM, Pickart CM. In vitro assembly and recognition of Lys-63 polyubiquitin chains. J Biol Chem 2001; 276:27936-27943.

28 Nuber U, Schwarz SE, Scheffner M. The ubiquitin-protein ligase E6-associated protein (E6-AP) serves as its own substrate. Eur J Biochem 1998; 254:643-649.

29 Yang Y, Fang S, Jensen JP, Weissman AM, Ashwell JD. Ubiquitin protein ligase activity of IAPs and their degradation in proteasomes in response to apoptotic stimuli. Science 2000; 288:874-877.

30 Bruce MC, Kanelis V, Fouladkou F, et al. Regulation of Nedd4-2 self-ubiquitination and stability by a PY motif located within its HECT-domain. Biochem J 2008; 415:155-163.

31 Lamothe B, Besse A, Campos AD, et al. Site-specific Lys-63linked tumor necrosis factor receptor-associated factor 6 autoubiquitination is a critical determinant of I $\mathrm{\kappa B}$ kinase activation. J Biol Chem 2007; 282:4102-4112.

32 Ben-Saadon R, Zaaroor D, Ziv T, Ciechanover A. The polycomb protein Ring1B generates self atypical mixed ubiquitin chains required for its in vitro histone $\mathrm{H} 2 \mathrm{~A}$ ligase activity. Mol Cell 2006; 24:701-711.

33 Xu HM, Liao B, Zhang QJ, et al. Wwp2, an E3 ubiquitin ligase that targets transcription factor Oct-4 for ubiquitination. J Biol Chem 2004; 279:23495-23503.

34 Pickart CM, Fushman D. Polyubiquitin chains: polymeric protein signals. Curr Opin Chem Biol 2004; 8:610-616.

35 Jin L, Williamson A, Banerjee S, Philipp I, Rape M. Mechanism of ubiquitin-chain formation by the human anaphasepromoting complex. Cell 2008; 133:653-665.

36 Hamazaki T, Oka M, Yamanaka S, Terada N. Aggregation of embryonic stem cells induces Nanog repression and primitive endoderm differentiation. J Cell Sci 2004; 117:5681-5686.

37 Miki K, Kitagawa Y. Biphasic response to retinoic acid dose in differentiation of F9 cells into primitive endoderm-like cells. Exp Cell Res 1988; 179:344-351.

38 Wiesner S, Ogunjimi AA, Wang HR, et al. Autoinhibition of the HECT-type ubiquitin ligase Smurf2 through its C2 domain. Cell 2007; 130:651-662.

39 Wu-Baer F, Lagrazon K, Yuan W, Baer R. The BRCA1/ BARD1 heterodimer assembles polyubiquitin chains through an unconventional linkage involving lysine residue K6 of ubiquitin. J Biol Chem 2003; 278:34743-34746.

40 Saeki Y, Kudo T, Sone T, et al. Lysine 63-linked polyubiquitin chain may serve as a targeting signal for the $26 \mathrm{~S}$ proteasome. EMBO J 2009; 28:359-371.

41 Oberst A, Malatesta M, Aqeilan RI, et al. The Nedd4-binding partner 1 (N4BP1) protein is an inhibitor of the E3 ligase Itch. Proc Natl Acad Sci USA 2007; 104:11280-11285.

42 Debonneville C, Flores SY, Kamynina E, et al. Phosphorylation of Nedd4-2 by Sgk1 regulates epithelial $\mathrm{Na}^{+}$channel cell surface expression. EMBO J 2001; 20:7052-7059.

43 Gao M, Labuda T, Xia Y, et al. Jun turnover is controlled through JNK-dependent phosphorylation of the E3 ligase Itch. 
Science 2004; 306:271-275.

44 Kee Y, Huibregtse JM. Regulation of catalytic activities of HECT ubiquitin ligases. Biochem Biophys Res Commun 2007; 354:329-333.

45 Woelk T, Oldrini B, Maspero E, et al. Molecular mechanisms of coupled monoubiquitination. Nat Cell Biol 2006; 8:12461254.

46 Pirozzi G, McConnell SJ, Uveges AJ, et al. Identification of novel human WW domain-containing proteins by cloning of ligand targets. J Biol Chem 1997; 272:14611-14616.

47 Ginis I, Luo Y, Miura T, et al. Differences between human and mouse embryonic stem cells. Dev Biol 2004; 269:360-
380.

48 Leng RP, Lin Y, Ma W, et al. Pirh2, a p53-induced ubiquitinprotein ligase, promotes p53 degradation. Cell 2003; 112:779791.

49 Li H, Zhang Z, Wang B, et al. Wwp2-mediated ubiquitination of the RNA polymerase II large subunit in mouse embryonic pluripotent stem cells. Mol Cell Biol 2007; 27:5296-5305.

50 Turksen K. Embryonic stem cells: Methods and Protocols. Totowa, NJ: Humana Press Inc, 2002.

51 Zhang Z, Liao B, Xu M, Jin Y. Post-translational modification of POU domain transcription factor Oct-4 by SUMO-1. FASEB J 2007; 21:3042-3051.

(Supplementary information is linked to the online version of the paper on the Cell Research website.) 